\title{
Primary Motor Cortex Asymmetry Is Correlated With Handedness in Capuchin Monkeys (Cebus apella)
}

\author{
Kimberley A. Phillips \\ Hiram College
}

\author{
Chet C. Sherwood \\ Kent State University
}

\begin{abstract}
Humans exhibit a population-wide tendency toward right-handedness, and structural asymmetries of the primary motor cortex are associated with hand preference. Reported are similar asymmetries correlated with hand preference in a New World monkey (Cebus apella) that does not display population-level handedness. Asymmetry of central sulcus depth is significantly different between left-handed and right-handed individuals as determined by a coordinated bimanual task. Left-handed individuals have a deeper central sulcus in the contralateral hemisphere; right-handed individuals have a more symmetrical central sulcus depth. Cerebral hemispheric specialization for hand preference is not uniquely human and may be more common among primates in general.
\end{abstract}

Keywords: brain asymmetry, handedness, Cebus, lateralization

Anatomical asymmetries of the human brain are thought to underlie the expression of lateralized functions such as hand preference and hemispheric language dominance (Toga \& Thompson, 2003). Although some studies have reported correlations between handedness and anatomical asymmetries of petalias and language-related cortical areas, asymmetry of the primary motor cortex may represent the most salient neurobiological substrate of hand preference (Hammond, 2002). The primary motor cortex is important for controlling kinematic aspects of movement, such as guiding the direction and amplitude of muscle forces that form successive movements (Geyer, Matelli, Luppino, \& Zilles, 2000; Lu \& Ashe, 2005). Horizontal interconnections among neurons in the primary motor cortex synchronize the spatiotemporal activity of different movement representations across dispersed cortical columns (Keller, 1993). In this context, anatomical studies in humans suggest that the primary motor cortex opposite to the dominant hand may have specializations of intrinsic circuitry. Histological data from postmortem human brains of unknown handedness indicate that there is a greater proportion of neuropil volume in the left hemisphere of the primary motor cortex hand representation area, suggesting the presence of more interconnectivity among neurons (Amunts et al., 1996). Furthermore, several studies have reported that asymmetry of the intrasulcal length of the precentral gyrus is associated with handedness (Amunts, Jancke, Mohlberg, Steinmetz, \& Zilles, 2000; Amunts et al., 1996; Foundas,

Kimberley A. Phillips, Departments of Psychology and Biology, Hiram College; Chet C. Sherwood, Department of Anthropology and School of Biomedical Sciences, Kent State University.

This work was supported by the National Science Foundation BCS0515484, Howard Hughes Medical Institute, the Wenner-Gren Foundation for Anthropological Research, Hiram College, and Kent State University. The American Psychological Association's guidelines concerning the ethical treatment of animals were followed during the course of this study. We thank our veterinary staff and the staff of the Brain Imaging Research Center for their assistance in the care of the animals during the magnetic resonance procedure.

Correspondence concerning this article should be addressed to Kimberley A. Phillips, Department of Psychology, Hiram College, P.O. Box 67, Hiram, OH 44234. E-mail: phillipsk@ hiram.edu
Hong, Leonard, \& Heilman, 1998; but see White et al., 1997). The most consistent finding arising from these studies is that the central sulcus in the region of hand representation is deeper in the dominant hemisphere of right-handed individuals, with more symmetrical central sulcus depth in left-handed individuals (Amunts et al., 2000; Foundas et al., 1998).

In contrast to these data from humans, the neurobiological basis of hand preference in nonhuman primates is poorly understood. Although there is evidence that captive chimpanzees are similar to humans in expressing a strong tendency toward population-level right-handedness (Hopkins \& Cantalupo, 2004; Hopkins, Wesley, Izard, Hook, \& Schapiro, 2004), most other primates do not display a directional hand preference at the population level (McGrew \& Marchant, 1997). Here we examine whether there is a correlation between asymmetry of the central sulcus and handedness in male, brown capuchin monkeys (Cebus apella). Monkeys of this species exhibit strong and consistent hand preferences during tasks requiring complex bimanual activity (e.g., Fragaszy \& Mitchell, 1990; Limongelli, Sonetti, \& Visalberghi, 1994; Westergaard \& Suomi, 1993a, 1993b, 1996), but not during various unimanual tasks (Spinozzi \& Truppa, 1999). Although lateral bias is not seen at the population level (Fragaszy, Visalberghi, \& Fedigan, 2004; McGrew \& Marchant, 1997), some evidence suggests that group-wide hand biases may exist. A comparison of monkey hand preferences in three groups revealed a group-wide bias for the left hand in tasks that required the use of fine spatial positioning (e.g., retrieving seeds from a crevice) and that had a strong haptic component (Fragaszy et al., 2004). However, Spinozzi, Castorina, and Truppa (1998) found a righthand bias among their group for a coordinated bimanual task. Therefore, this species provides a unique opportunity to investigate the relationship between intraspecific variation in handedness and brain asymmetry.

\section{Method}

\section{Subjects}

Seven male capuchin monkeys (Cebus apella) housed at the Hiram College capuchin laboratory (Hiram, Ohio) served as subjects for this 
study. All of the monkeys were socially housed in large indoor enclosures (either $2.1 \mathrm{~m} \times 2.4 \mathrm{~m} \times 1.7 \mathrm{~m}$ or $1.8 \mathrm{~m} \times 2.4 \mathrm{~m} \times 1.9 \mathrm{~m}$ ) enriched with perches, swings, and fresh browse. Monkeys ranged in age from 1-18 years $(M=7.00$ years, $S D=6.03)$. All monkeys were born in captivity and had been socially housed since their birth.

\section{Simple Reaching Task}

Hand preference was assessed using two tasks: a simple reaching task (unimanual) and a coordinated task. During the simple reaching task, a raisin was tossed to a spot in front of the monkey at a distance that required movement of all four limbs to reach. The hand used to retrieve the raisin was recorded as left or right. Each raisin was tossed to a location that required the monkey to assume a new position between trials.

\section{Coordinated Bimanual Task}

Hand preference was assessed using a coordinated bimanual action test known as the tube task (Hopkins, 1995). Each monkey was presented with a $6 \mathrm{~cm}$ long, $1.5 \mathrm{~cm}$ diameter piece of polyvinyl chloride pipe that had peanut butter smeared inside. To remove the food, monkeys had to hold the tube in one hand and use the other hand to retrieve the peanut butter. The hand used to retrieve food from inside the tube was recorded as left or right. Every instance in which a monkey inserted a hand into the tube, retrieved peanut butter, and brought the hand to the mouth was recorded. Data were recorded until the monkey lost interest in the tube, as indicated by discarding the tube for at least $10 \mathrm{~s}$. Each monkey was tested 4 times with at least 2 days intervening between testing sessions.

\section{Behavioral Assessment}

Hand preference for each monkey in each task was determined by calculating the handedness index (HI) using the formula (\#R - \#L)/(\#R + $\# \mathrm{~L}$ ), where \#R is the number of instances in which the right hand was used, and \#L equals the number of instances in which the left hand was used. The $\mathrm{HI}$ produces scores ranging from -1 to +1 , with negative scores indicating a preference for the left hand and positive scores indicating a preference for the right hand. More extreme absolute scores reflect a stronger preference for a given hand. HI scores were calculated for the simple reaching task, each tube task trial, and the mean $\mathrm{HI}$ for all tube task trials. To determine if the hand preference of a monkey was significant, $z$ scores were calculated for the simple reaching task and mean $\mathrm{HI}$ for tube trials. Subjects with $z$ scores greater than 1.95 or less than -1.95 were classified as unambiguously right- or left-handed. Monkeys with $z$ scores between 1.95 and -1.95 were classified as having no hand preference.

\section{Magnetic Resonance Imaging (MRI) Procedure}

Monkeys were transported to the Brain Imaging Research Center in Pittsburgh, Pennsylvania, for the MRI procedure. At the facility, monkeys were anesthetized with ketamine $(25 \mathrm{mg} / \mathrm{kg})$ and acetylpromazine $(1 \mathrm{mg} /$ $\mathrm{kg})$ injection intramuscularly; atropine $(0.05 \mathrm{mg} / \mathrm{kg})$ was given subcutaneously. A constant intravenous drip of $160-330 \mu \mathrm{g} / \mathrm{kg} / \mathrm{min}$ of propofol was administered via intravenous catheter in the saphenous vein to maintain anesthesia. Monkeys were placed into the scanner chamber, and their heads were fitted inside a 16-in. head coil. Monkeys remained anesthetized during the MRI procedure. While anesthetized, respiration rate, heart rate, and oxygen saturation were continually monitored. Monkeys were allowed to completely recover from the effects of the anesthesia before being transported back to Hiram College.

MRIs of brains were acquired on a Siemens 3T Allegra Scanner. Sagittal T1-weighted 3D MPRAGE MR images were acquired through the entire brain using a $\mathrm{TR}=1,500 \mathrm{~ms}$ and $\mathrm{TE}=3.04 \mathrm{~ms}$ with no echo-train. Scan acquisition time was approximately $20 \mathrm{~min}$. For each monkey the number of signals averaged three. Slices were obtained as 0.5 -mm thick contiguous sections with a matrix size of $256 \times 256$ and a field of view of $128 \mathrm{~mm} \times$ $128 \mathrm{~mm}$, resulting in a final voxel size of $0.5 \mathrm{~mm} \times 0.5 \mathrm{~mm} \times 0.5 \mathrm{~mm}$. Images were spatially realigned into standard anatomical orientation with the transaxial plane parallel to the anterior commissure-posterior commissure line and perpendicular to the interhemispheric fissure. Prior to morphometric analysis, data were converted into the ANALYZE 3D volume file format to facilitate reslicing into orthogonal planes. Computer files for individual monkeys were numerically coded prior to measurement to prevent observer bias.

\section{Image Quantification Method}

Morphometric measurements were performed using ImageJ software version 1.26t (http://rsb.info.nih.gov/ij/). Central sulcus depth was measured as the intrasulcal length of the precentral gyrus as viewed in horizontal sections. Only the portion of the precentral gyrus representing the hand was selected for measurement because asymmetries in central sulcus depth at this location have previously been shown to correlate significantly with handedness in human males, whereas other regions do not (Amunts et al., 2000). The topographic location of hand representation on the motor strip was approximated on the basis of previous electrophysiological mapping studies in Cebus monkeys (Asanuma \& Rosen, 1972; Shinoda, Zarzecki, \& Asanuma, 1979). This region of interest corresponds to the series of sections located between $40 \%$ and $60 \%$ of the total dorsoventral length of the central sulcus as measured starting at the first dorsal section containing the central sulcus. This procedure resulted in measurement of an average of $6.1(S D= \pm 0.9)$ sections in each monkey. In each section, the intrasulcal length of the precentral gyrus was manually traced from both hemispheres. The asymmetry quotient (AQ; $\{\mathrm{R}-\mathrm{L} /[(\mathrm{R}+\mathrm{L}) * 0.5]\} *$ 100) of central sulcus depth was then calculated for each section. Finally, a summary AQ for the region of hand representation was calculated as the mean of all AQ measurements for all sections. Interobserver reliability (interclass correlation [ICC]) of the mean central sulcus AQ measure was ICC $=0.81, p<.05$ (observers were CCS and KAP). KAP's measurements were used in the analyses.

\section{Results}

Monkeys performed a mean of $52.71(S D= \pm 3.86)$ simple reaching acts and $34.14(S D= \pm 17.93)$ hand actions per tube task trial. Simple reaching HI, coordinated bimanual $M \mathrm{HI}$, and central sulcus AQ for each monkey are shown in Table 1 and Figure 1. The test-retest correlation for the coordinated bimanual HI trials was $r=0.77, p<.01$, showing that hand preference in this task is stable within monkeys across time.

Only 2 out of 7 monkeys exhibited a strong unambiguous preference to use one hand as dominant in the simple reaching task, as determined by a $z$ score for HI exceeding 1.95 or -1.95 . Both of these monkeys displayed a left-hand preference. The remaining 5 monkeys displayed no hand preference in the simple reaching task. In contrast, 6 monkeys expressed strong hand dominance in the coordinated bimanual task. Three monkeys displayed a left-hand preference, 3 monkeys displayed a right-hand preference, and 1 monkey displayed no hand preference. Simple reaching HI did not correlate with central sulcus AQ $\left(r_{s}=.04, p=.94\right)$ or with coordinated bimanual $M \mathrm{HI}\left(r_{s}=.07, p=.88\right)$ and so was not used in further analyses.

We examined whether central sulcus AQs were associated with handedness in the coordinated bimanual task. When divided into dichotomous groups according to hand preference, central sulcus AQ was significantly different $(U[3,3]=0, p=.05)$ between 
Table 1

Simple Reaching Handedness Index (HI), Tube Task Mean HI, and Asymmetry Quotients (AQ) for Central Sulcus Depth for Each Monkey

\begin{tabular}{rcccrrr}
\hline & \multicolumn{3}{c}{ Simple } & & \multicolumn{2}{c}{ Central sulcus } \\
Monkey ID & Age (in years) & reaching HI & Simple reaching $z$ & Tube task $M$ HI & Tube task $z$ & AQ \\
\hline CA003 & 18 & -0.84 & 6.52 & -1.00 & -14.59 & 10.80 \\
H03 & 2.5 & 0.18 & -1.29 & 0.81 & 7.40 & 0.90 \\
CA9207 & 12 & 0.02 & -0.14 & 1.00 & 7.02 & -3.00 \\
H04 & 1.5 & -0.61 & 4.34 & -0.14 & -0.68 & -2.60 \\
CG29 & 5 & 0.25 & -1.79 & -0.95 & -8.61 & 7.00 \\
H02 & 3.5 & 0.20 & 0.28 & -0.62 & -8.64 & 6.00 \\
H06 & 1 & -0.12 & 0.83 & 0.39 & 10.44 & -0.20 \\
\hline
\end{tabular}

Note. Negative HI scores indicate a preference for the left hand, and positive HI scores indicate a preference for the right hand. For the central sulcus AQ, a positive AQ indicates right-hemisphere dominance, a negative AQ indicates left-hemisphere dominance. ID = identification.

left-handed $(M=7.93, S E=1.47, n=3)$ and right-handed monkeys $(M=-0.76, S E=1.15, n=3)$. Next, we tested whether there was a correlation between continuous variables representing
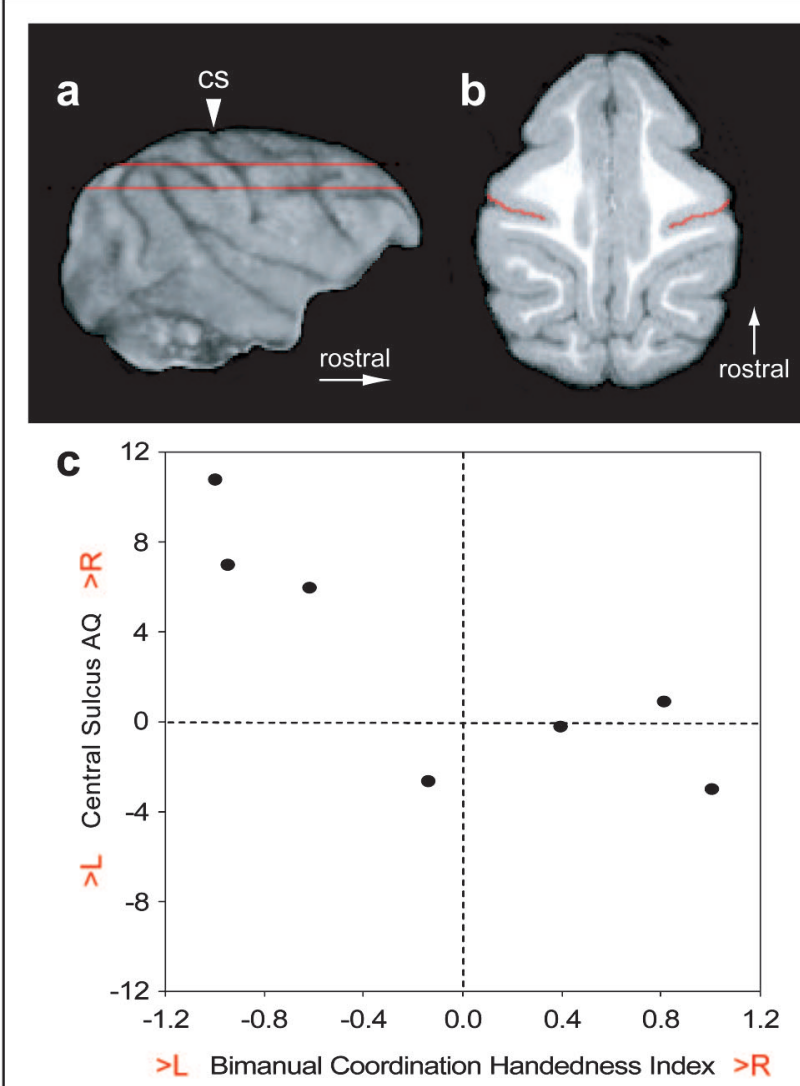

Figure 1. a. Horizontal lines indicate the region of sampling for hand representation ( $\mathrm{cs}=$ central sulcus). b. CS depth was measured as the intrasulcal length of the precentral gyrus as viewed in horizontal sections. c. Individual asymmetry quotients (AQ; $\{\mathrm{R}-\mathrm{L} /[(\mathrm{R}+\mathrm{L}) * 0.5]\} * 100$ ), where $\mathrm{R}$ and $\mathrm{L}$ represent $\mathrm{CS}$ depth in the right and left hemisphere, respectively. The sign of the AQ indicates the direction of asymmetry: A positive AQ indicates right-hemisphere asymmetry, a negative AQ indicates left-hemisphere asymmetry. The AQ of CS depth is significantly different between left- and right-handed monkeys $(U[3,3]=0, p=.05)$. the direction and degree of brain asymmetry and handedness. We found a significant negative correlation between central sulcus AQ and coordinated bimanual $\mathrm{MHI}\left(r_{s}[7]=-.857, p<.02\right.$; see Figure 1c).

Taken together, these findings indicate that there is only a significant association between central sulcus asymmetry and lateralization in the coordinated bimanual task, not simple reaching. Furthermore, monkeys that showed a left-hand bias in the coordinated bimanual task displayed a deeper central sulcus on the right, whereas the central sulcus was more symmetrical in right-handed monkeys. Finally, we examined central sulcus AQ values for population-level asymmetry by using a one-sample $t$ test. Whereas the result did not reach statistical significance for greater central sulcus depth on the right side, $t(6)=1.353, p=.22$, the difference was in the expected direction.

\section{Discussion}

The results of this study suggest that there might be an underlying right-hemisphere bias in the primary motor cortex of capuchins, as central sulcus asymmetries were most pronounced in monkeys that were left-hand dominant. Given the absence of population-level hand preference in capuchins (Fragaszy et al., 2004; McGrew \& Marchant, 1997), it is noteworthy that a cortical area linked with handedness in humans is also related to hand dominance in capuchins. It is also interesting that the correlation between handedness and asymmetry of the primary motor cortex was not generalized to the simple reaching task, but instead was restricted to bimanual coordination. This result is consistent with the finding that only the tube task is significantly correlated with asymmetry of the precentral gyrus in chimpanzees (Hopkins \& Cantalupo, 2004). In this task, the dominant hand performs sequences of manipulative actions, whereas the subordinate hand is kept in a fixed posture to stabilize the object. It is possible that the circuitry within the primary motor cortex necessary to perform such sequences of synchronized manual actions by the dominant hand requires elaboration of neuron numbers and/or dendritic arbors, thereby causing a gross anatomical lengthening of the surface of the precentral gyrus. Such anatomical specializations may not be necessary to subserve lateralization of a relatively uncomplicated action such as reaching.

The association between handedness for the bimanual task and central sulcus morphology in capuchins, however, is the mirror re- 
verse of findings in humans. In human males, central sulcus depth is greater in the contralateral hemisphere of right-handed individuals and asymmetries decrease in mixed- and left-handers (Amunts et al., 2000). The same study, however, did not find a significant correlation between handedness and central sulcus asymmetry in females, suggesting sex differences in cortical control of hand movements.

Although some theories have linked population-level righthandedness to hemispheric language dominance in humans (e.g., Annett, 2002), recent evidence from captive chimpanzees indicates that hand preference is correlated with asymmetries of the primary motor cortex but not language area homologues (Hopkins \& Cantalupo, 2004). Taken together with our results, it appears that hemispheric specialization for hand preference may be common among primates and may be unrelated to language. However, the differences between capuchins and humans in the direction of hemispheric bias of the central sulcus and its relationship to population-level handedness suggest that distinct genetic and neurobiological mechanisms underlie handedness in these groups.

To more fully explore the relationship between manipulative abilities, handedness, and central sulcus morphology among primates, future studies should be directed at collecting similar correlative brain and behavioral data from a greater number of species, including both sexes. Previous work on lateralization in primates has focused mostly on examining population-level asymmetries of hand preference or brain asymmetry separately. Our results indicate a potential relationship between variation in behavioral laterality and neuroanatomical asymmetry within a species. It is possible that this relationship applies to other species that also lack population-level effects. In many prosimians and monkeys, the left hand is favored for visually guided reaching, whereas the right hand is used for postural support (MacNeilage, Studdert-Kennedy, \& Lindblom, 1987). Thus, we speculate that central sulcus asymmetry in capuchins might be related to righthemispheric specialization for motor integration of visuospatial information in the context of complex feeding actions. It will be important to examine whether a similar pattern of right-hemispheric dominant asymmetry is present in other prosimian and monkey species. It will be also be essential to extend future studies to include larger samples of capuchins, including females, to determine whether human-like sex differences in the cortical organization of hand control are present. Our current findings, along with these comparative data, will provide a necessary foundation to trace the evolution of handedness in humans and other primates.

\section{References}

Amunts, K., Jäncke, L., Mohlberg, H., Steinmetz, H., \& Zilles, K. (2000). Interhemispheric asymmetry of the human motor cortex related to handedness and gender. Neuropsychologia, 38, 304-312.

Amunts, K., Schlaug, G., Schleicher, A., Steinmetz, H., Dabringhaus, A., Roland, P. E., \& Zilles, K. (1996). Asymmetry in the human motor cortex and handedness. Neuroimage, 4, 216-222.

Annett, M. (2002). Handedness and brain asymmetry: The right shift theory. Hove, UK: Psychology Press.

Asanuma, H., \& Rosen, I. (1972). Topographical organization of cortical efferent zones projecting to distal forelimb muscles in the monkey. Experimental Brain Research, 14, 243-256.

Foundas, A. L., Hong, K., Leonard, C. M., \& Heilman, K. M. (1998). Hand preference and magnetic resonance imaging asymmetries of the central sulcus. Neuropsychiatry, Neuropsychology, and Behavioral Neurology, $11(2), 65-71$
Fragaszy, D. M., \& Mitchell, S. R. (1990). Hand preference and performance on unimanual and bimanual tasks in capuchin monkeys (Cebus apella). Journal of Comparative Psychology, 104, 272-282.

Fragaszy, D. M., Visalberghi, E., \& Fedigan, L. M. (2004). The complete capuchin. Cambridge, UK: Cambridge University Press.

Geyer, S., Matelli, M., Luppino, G., \& Zilles, K. (2000). Functional neuroanatomy of the primate isocortical motor system. Anatomy and Embryology, 202, 443-474.

Hammond, G. (2002). Correlates of human handedness in primary motor cortex: A review and hypothesis. Neuroscience \& Biobehavioral Reviews, 26, 285-292.

Hopkins, W. D. (1995). Hand preferences for a coordinated bimanual task in 110 chimpanzees: Cross-sectional analysis. Journal of Comparative Psychology, 109, 291-297.

Hopkins, W. D., \& Cantalupo, C. (2004). Handedness in chimpanzees (Pan troglodytes) is associated with asymmetries of the primary motor cortex but not with homologous language areas. Behavioral Neuroscience, 118, $1176-1183$

Hopkins, W. D., Wesley, M. J., Izard, M. K., Hook, M., \& Schapiro, S. J. (2004). Chimpanzees (Pan troglodytes) are predominantly right-handed: Replication in three populations of apes. Behavioral Neuroscience, 118, $659-663$

Keller, A. (1993). Intrinsic synaptic organization of the motor cortex Cerebral Cortex, 3, 430-441.

Limongelli, L., Sonetti, M. G., \& Visalberghi, E. (1994). Hand preference of tufted capuchins (Cebus apella) in tool-using tasks. In J. R. Anderson, J. J. Roeder, B. Thierry, \& N. Hammerschmidt (Eds.), Current primatology (pp. 9-15). Strasbourg, France: University Louis Pasteur Press.

Lu, X., \& Ashe, J. (2005). Anticipatory activity in primary motor cortex codes memorized movement sequences. Neuron, 45, 967-973.

MacNeilage, P. F., Studdert-Kennedy, M. G., \& Lindblom, B. (1987). Primate handedness reconsidered. Behavioral \& Brain Sciences, 10, 247-303.

McGrew, W. C., \& Marchant, L. F. (1997). On the other hand: Current issues in and meta-analysis of the behavioral laterality of hand function in nonhuman primates. Yearbook of Physical Anthropology, 40, 201232.

Shinoda, Y., Zarzecki, P., \& Asanuma, H. (1979). Spinal branching of pyramidal tract neurons in the monkey. Experimental Brain Research, $34,59-72$.

Spinozzi, G., Castorina, M. G., \& Truppa, V. (1998). Hand preferences in unimanual and coordinated-bimanual tasks by tufted capuchin monkeys (Cebus apella), Journal of Comparative Psychology, 112, 183-191.

Spinozzi, G., \& Truppa, V. (1999). Hand preferences in different tasks by tufted capuchins (Cebus apella). International Journal of Primatology, $20,827-849$

Toga, A. W., \& Thompson, P. M. (2003). Mapping brain asymmetry. Nature Reviews Neuroscience, 4(1), 37-48.

Westergaard, G. C., \& Suomi, S. J. (1993a). Hand preference in capuchin monkeys (Cebus apella) varies with age. Primates, 34, 295-299.

Westergaard, G. C., \& Suomi, S. J. (1993b). Hand preference in the use of nut-cracking tools by tufted capuchin monkeys (Cebus apella). Folia Primatologica, 61, 38-42.

Westergaard, G. C., \& Suomi, S. J. (1996). Hand preference for a bimanual task in tufted capuchins (Cebus apella) and rhesus macaques (Macaca mulatta). Journal of Comparative Psychology, 110, 406-411.

White, L. E., Andrews, T. J., Hulette, C., Richards, A., Groelle, M., Paydarfar, J., et al. (1997). Structure of the human sensorimotor system. II: Lateral symmetry. Cerebral Cortex, 7, 31-47.

Received June 1, 2005

Revision received August 8, 2005

Accepted August 17, 2005 